\title{
Einfluss der Produktreife auf die Bewertung der Anwendungseignung technischer Produkte
}

Kamil Trojan ${ }^{1}$, René Germann ${ }^{1}$, Sven Matthiesen ${ }^{1}$

${ }^{1}$ Institute of Product Engineering (IPEK),

Karlsruhe Institute of Technology (KIT), Germany

\section{Abstract}

For the success of a technical product, it must be perceived by the user as suitable as possible for its use. In the early phase of product development, prototypes are used to validate the perceived suitability for use. They differ in their prototype fidelity through appearance and haptics and they influence the users' perception of suitability for use. Due to lack of knowledge, no generalizable effect of the prototype fidelity is available yet. In the presented research project, a reduction approach is investigated with the aim to build up an understanding for the influences on the subjective suitability for use, and thus to enable an objective assessment. This work demonstrates the approach through a study design conducted with Power-Tools and test subjects.

Keywords: Prototype fidelity, Usability testing, Ergonomics, Aesthetics

\section{Einleitung}

Die Einbeziehung des potentiellen Anwenders in die Anforderungsermittlung für ein neues technisches Produkt ist unverzichtbar, um ein erfolgreiches Produkt entwickeln zu können [1]. Mit der nutzerzentrierten Produktentwicklung wird daher versucht, die Anwenderbedürfnisse, welche oft nur unterbewusst vorhanden sind, zu berücksichtigen und Produkte so zu konzipieren, dass 
sie zu den Bedürfnissen des Anwenders passen. In Folge solcher Optimierungen kann es nachweislich zur Zunahme der Produktivität kommen, da der Kunde seine Anwenderbedürfnisse als erfüllt bewertet [2]. Diese subjektiv geprägte Wahrnehmung des Anwenders in Interaktion mit einem Produkt kann in einem Produkttest zusammen mit dem Anwender stattfinden. Hierbei ist zu beachten, dass die Festlegung auf bestimmte zu optimierende Produkteigenschaften schon in der frühen Phase der Produktentwicklung stattfindet, in welcher oft noch keine Serienprodukte für einen Test zur Verfügung stehen. Diesem Problem wird mit prototypischen Fertigungsverfahren, zum Beispiel dem additiven Fertigungsverfahren oder auch 3D gedruckten Teilen begegnet [3]. Durch den sich von der Serie unterscheidenden Produktreifegrad kommt es hierbei zu dem Problem, dass objektive Aussagen zur Produkteignung und der wahrgenommenen Anwendungseignung nur bedingt möglich sind. Dies liegt nach Aussage mehrerer Forscher hauptsächlich daran, dass ein Produkt-Prototyp eine geänderte Wahrnehmung beim Anwender erzeugt $[4,5,6,7]$ und damit anstatt der gewünschten Produkteigenschaften der Einfluss von prototypischen Eigenschaften bewertet wird. Zur Berücksichtigung dieses Problems gibt es im aktuellen Stand der Forschung nur wenige Forschungsarbeiten, welche sich mit dieser Problematik beschäftigen. Um dieses Problem zu adressieren, wird innerhalb dieses Beitrages ein geplantes Forschungsvorhaben vorgestellt, welches das Ziel hat, den Einfluss der Produktreife auf die Bewertung der wahrgenommenen Anwendungseignung zu untersuchen und darauf basierend Ansätze zu entwickeln, welche eine Berücksichtigung dieses Einflusses innerhalb des Produktentwicklungsprozesses ermöglichen.

\section{Stand der Forschung}

\subsection{Anwendungseignung}

Werden Produktbewertungen durchgeführt, bewertet der Anwender innerhalb eines Produkttests in einer definierten Anwendung sein subjektives Qualitätsempfinden, welches stark von der durchgeführten Anwendung und dem bewertenden Anwender abhängig ist. MATTHIESEN UND GERMANN nutzen zur Beschreibung dieses subjektiven Qualitätsempfindens in der Anwendung den Begriff der Anwendungsqualität [8]. Für die Anwendungsqualität spielen zwei Faktoren eine Rolle - die objektive technische Funktionsfähigkeit und die subjektiv wahrgenommene Funktionalität. Die objektive technische Funktionsfähigkeit kann der Produktentwickler direkt und bewusst beeinflussen. Die subjektiv wahrgenommene Funktionalität in der Anwendung wird sowohl von Aspekten der Gebrauchstauglichkeit, als auch der User Experience beeinflusst, welche sich erst während der Nutzung eines Produkts entwickeln und je nach Anwendung und Anwender unterschiedlich wahrgenommen werden [9]. MATTHIESEN 
UND GERMANN fassen dabei die Einflüsse beider Aspekte zusammen und definieren den Begriff der wahrgenommenen Anwendungseignung eines Produktes wie folgt: "Subjektiv wahrgenommenes Ausmaß, wie effektiv, effizient und zufriedenstellend mit einem technischen Produkt, durch einen definierten Anwender, relevante Anwendungen zielführend durchgeführt werden können" [8].

\subsection{Erfassung von Usability-Aspekten und Störgrößen}

Die Erfassung der wahrgenommenen Anwendungseignung kann mit Methoden aus dem Anforderungsmanagement (z.B. Fragebogen, Interview, Beobachtung) stattfinden. Die Erfassung der Bewertung des Anwenders wird begleitet von dem Problem der Subjektivität der Wahrnehmung, die wiederum von unterschiedlichen Aspekten beeinflusst werden kann. Diese nach GERMANN ET AL. als Usability-Aspekte bezeichneten Einflüsse [4] können von physischer, physiologischer oder psychologische, sozialer oder kultureller Natur sein und beeinflussen sich stark gegenseitig [10]. Auch in der DIN EN ISO 9241220:2017-05 werden die physikalischen, sozialen und kulturellen Aspekte aufgeführt, die Einfluss auf den Nutzungskontext und damit auf die wahrgenommene Anwendungseignung haben [9]. Je nach Ausrichtung des Entwicklungsfokus, sollen manche Usability-Aspekte, wie beispielsweise Vorurteile, Emotionen oder Erfahrungswissen des Anwenders nicht innerhalb der Produktbewertung berücksichtigt werden [11]. Eine Abgrenzung oder Berücksichtigung dieser störenden Usability-Aspekte innerhalb der Probandenbewertung ist jedoch nicht immer möglich und es kommt zu verzerrenden Effekten der wahrgenommenen Anwendungseignung, da diese teilweise einen signifikanten Einfluss auf die Wahrnehmung des Anwenders ausüben [11]. Diese als Störgrößen bezeichneten Einflüsse sind bei der Erfassung unerwünscht und stellen für die Produktentwicklung ein Problem dar und können diese fehlleiten [4].

\subsection{Prototypen in der Produktentwicklung}

Zur Untersuchung der Anwendungseignung in der frühen Phase der Produktentwicklung werden Prototypen eingesetzt [3]. Einige Forschungsarbeiten bestätigen die positiven Effekte von Prototypen im Produktentstehungsprozess $[12,13]$. Das Prototyping ist bei vielen Entwicklern ein fester Bestandteil und wird je nach Zielstellung, Einsatzweck oder erforderlicher Charakteristik unterschiedlich eingesetzt [14]. Oft werden Prototypen mit reduzierter Produktreife eingesetzt (Papierprototyp, 3-D-Druck u.ä.), da sie günstiger und verfügbarer in der frühen Phase der Produktentwicklung sind [14]. Diese Prototypen unterscheiden sich ihrer Produktreife von der Serie durch ihre Fertigungsart, in der Ästhetik und ebenso in der Haptik. Zudem besitzen sie meist eine reduzierte Funktionsbreite. Nach VIRZI ET AL. kann eine Klassifizierung der Produktreife 
(engl. Prototype fidelty) in vielen unterschiedlichen Aspekten relativ zum finalen Produkt stattfinden [8]. Diese Aspekte können zum Beispiel die Funktionsbreite (Anzahl Funktionen), der Grad an Funktionalität, die Gleichartigkeit der Anwendungsinteraktion (Kraftaufwand bei der Betätigung eines Schalters) oder die ästhetische Differenz (Farben, grafisches Design) sein [8]. Eine Einschränkung ist jedoch, dass der Einsatz von Prototypen mit reduzierter Produktreife mit einem weniger genauen Eindruck der Anwenderbewertung einhergeht [15]. Weitere Arbeiten dokumentieren ein verändertes Nutzerverhalten bei der Interaktion mit reduzierter Produktreife und eine veränderte, störgrößenbehaftete Bewertung [16].

\subsection{Störgröße Produktreife bei der Erfassung von Usability-Aspekten}

Für die nutzerzentrierte Produktentwicklung werden objektiv erfasste Entwicklungsziele, welche nicht durch den Einfluss von Störgrößen verzerrt sind, benötigt. Neben den bereits in Kapitel 2.2 genannten Störgrößen konnten GERMANN ET AL. $[4,17]$ in mehreren Arbeiten zur Bewertung von Griffgeometrien von Akkuschraubern den Einfluss der Störgrößen Erfahrungswissen und Marke auf die subjektive Anwendungseignung nachweisen, wobei durch den Einsatz von Reduktionsansätzen der Einfluss der Störgrößen signifikant reduziert werden konnte. Weiterhin konnten die Forschungsarbeiten zeigen, dass der Aspekt der "Produktreife" auf die Bewertung der Anwendungseignung einen erheblichen Einfluss ausübt und daher berücksichtigt werden sollte [4]. Als mögliche Gründe werden unter anderem die Fertigungsart, das Aussehen und die taktile Wahrnehmung der Prototypen gesehen. Dazu konnten JENSEN ET AL. am Beispiel eines Vorhängeschlosses und SAUER UND SONDEREGGER am Beispiel von Mobiltelefonen nachweisen, dass die Produktreife signifikant durch die Variation der Teilaspekte der Ästhetik und der Haptik hinsichtlich der Bewertung des Nutzers beeinflusst werden kann $[7,16]$. Es wurden sowohl die Ästhetik als auch die Fertigungsarten der Prototypen variiert, wobei dem Aspekt der Ästhetik durch eine Modifizierung der Farbe und des Musters ein signifikanter Einfluss auf die Anwendungseignung und die Attraktivität zugesprochen wurde. Dieser Einfluss ist nach JENSEN ET AL. sehr komplex und nicht linear verlaufend [7], was weiteren Forschungsbedarf zum Aufbau des Verständnisses zum Einfluss der Produktreife notwendig macht $[7,16]$. Insgesamt gibt es nur wenige Studien zum Störgrößeneinfluss der Produktreife $[6,7,15,16]$ und Aussagen zu generalisierbaren Auswirkungen einer reduzierten Produktreife auf die Bewertungen durch Anwender sind auf Grund von fehlenden Erkenntnissen nicht möglich. Des Weiteren liegen im aktuellen Stand der Forschung keine Ansätze zur Reduktion des Störgrößeneinflusses der Produktreife auf die Bewertung der wahrgenommenen Anwendungseignung vor, wie sie zum Beispiel für den Einfluss der Marke oder der Expertise in der Anwendung entwickelt wurden. 


\section{Forschungsziel}

Mit den aus dem Stand der Forschung aufgezeigten Ansätzen ist eine Berücksichtigung der Störgröße „Produktreife" bisher nur eingeschränkt möglich. Aus dem Stand der Forschung kann jedoch der erhebliche Einfluss der Störgröße der Produktreife auf die wahrgenommene Anwendungseignung aufgezeigt werden. Der Einfluss der Produktreife auf die Bewertung der wahrgenommenen Anwendungseignung, wird daher im Folgenden unter Berücksichtigung der Aspekte der Ästhetik und der Haptik analysiert. Ziel dieses Beitrages ist die Vorstellung des Vorgehens zur Erforschung des Einflusses der Produktreife auf die wahrgenommene Anwendungseignung und die Übertragung des aufgebauten Wissens in für die Produktentwicklung nutzbare methodische Ansätze. Zur Erreichung des Forschungsziels ist dieses in mehrere Forschungsfragen aufgeteilt (Bild 1), die innerhalb von einzelnen Studien beantwortet werden.

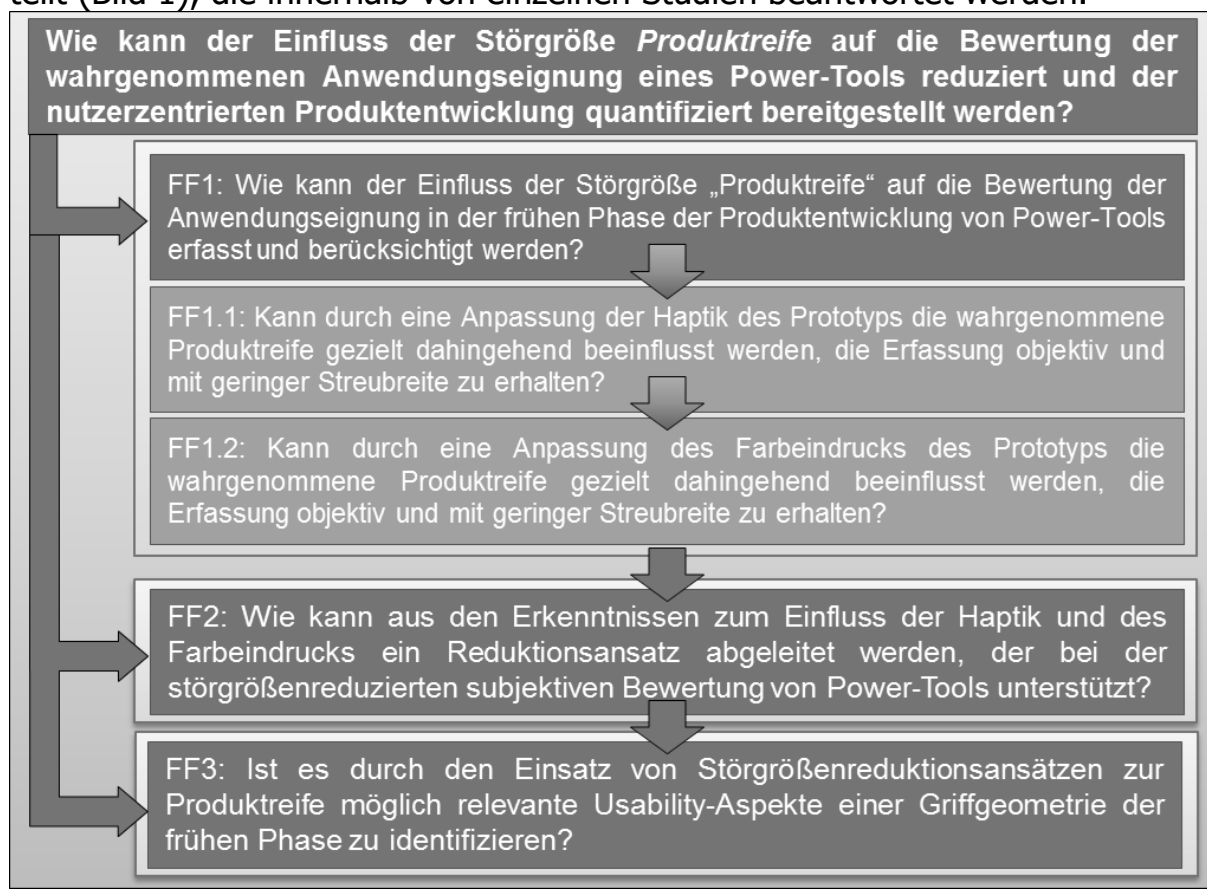

Bild 1 - Forschungsfragen für das Forschungsvorhaben

\section{Forschungsdesign}

Um die Störgröße "Produktreife" zu erforschen und einen Reduktionsansatz entwickeln zu können, werden drei Probandenstudien mit professionellen Anwendern und Power-Tools durchgeführt. Das allgemeine Studiendesign, dass 
die Beantwortung der Forschungsfragen ermöglichen soll, wird hier am Beispiel der ersten Probandenstudie zur Beantwortung von FF1 vorgestellt. Innerhalb dieser wird die wahrgenommene Anwendungseignung von Power-Tool-Prototypen in definierten, realitätsnahen, kontrollierten Testfällen bewertet werden. Die erste Studie wird am Beispiel eines Akkuschraubers durchgeführt. Für diesen werden mehrere Prototypen hergestellt. Die Prototypen werden in ihren haptischen und ästhetischen Eigenschaften fertigungstechnisch und optisch variiert. Als Referenzbewertung dient der gleiche Akkuschrauber aus der Serienfertigung. Durch das vorgestellte Vorgehen wird Forschungsfrage 1.1/1.2 mit statistischen Ansätzen analysiert und erste Erkenntnisse in die Herleitung eines initialen Reduktionsansatzes einfließen. Zur Beantwortung der FF2 und zur Weiterentwicklung des initial entworfenen Reduktionsansatzes wird am Beispiel eines Bohrhammers eine Studie aufgebaut. Dieser Power-Tool-Typ unterscheidet sich vom Akkuschrauber durch die Führung mit beiden Händen, wodurch der Anwender noch stärker im Leistungs- und Informationsfluss während der Anwendung steht. Um zu überprüfen, ob der durch die Erkenntnisse aus Studie 1 und 2 entwickelte Reduktionsansatz wirksam angewendet werden kann, wird dieser am Beispiel einer Griffgeometrieoptimierung am Akkuschrauber validiert. Hierfür wird ein Prototyp mit leicht auswechselbaren Griffstückschalen entwickelt und aufgebaut. Ziel der Untersuchung ist die Identifikation relevanter Usability-Aspekte, welche gezielt innerhalb der Produktentwicklung auf den jeweiligen Anwendungsfall angepasst werden können (FF3). Im Folgenden wird anhand der im Forschungsdesign geplanten Untersuchung zur Beantwortung der Forschungsfrage F1.1 / F1.2 das geplante Studiendesign vorgestellt, welches die Grundlage für die weiteren Studien im Forschungsvorhaben darstellt.

\subsection{Vorstellung des Studiendesigns}

Bei der Erforschung des Einflusses von Störgrößen ist es notwendig, die unabhängigen Variablen aus der Anwendungsumgebung zu identifizieren, einzustellen und ihren Effekt auf geeignete abhängigen Variablen zu erfassen. Im konkreten Beispiel der ersten Studie dieses Forschungsvorhabens werden die unabhängigen Variablen Farbkombination, $\mathrm{R}_{\mathrm{z}}$-Wert und Sichtbarkeit des Fertigungsverfahrens identifiziert und variiert. Die abhängigen Variablen wahrgenommene Anwendungseignung, Griffkomfort, Funktionalität und Farbeindruck werden durch den Anwender bewertet (s. Bild 2). 


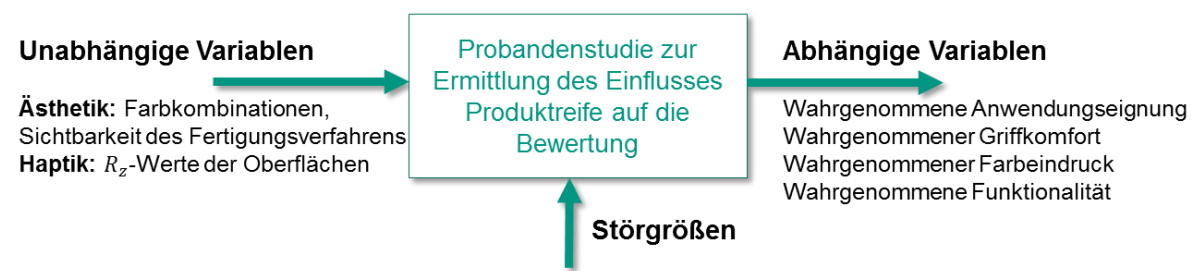

Bild 2 - Unabhängige und abhängige Variablen in Studie 1

\subsection{Versuchsgerät}

Als Demonstrator zur Bewertung der Usability-Aspekte wird ein Profi-Akkuschrauber als Power-Tool verwendet. Dieser ist für den täglichen Einsatz geeignet und kann nach Vorerprobungen ohne Verschleißerscheinung mindestens $30 \mathrm{~h}$ eingesetzt verwendet werden. Das sichert die Studiendurchführung gegen technische Ausfälle ab. Als Referenz-Power-Tool zur Bewertung im Vergleich zu Prototypen reduzierter Produktreife dient das Seriengerät, welches anonymisiert wurde, um den Einfluss durch die Marke zu vermeiden. Der schwarze Kunststoff weist eine gemittelte Rauigkeit mit dem Rz-Wert von 19,33 um und die graue Gummierung einen $R_{z}$-Wert von $18,38 \mu \mathrm{m}$ auf. Um eine sich von der Serie unterscheidende Produktreife nach VIRZI ET AL. zu erhalten [6], wird das Gehäuse des Power-Tools aus dem Werkstoff ABS additiv im FDM-Verfahren hergestellt und in Teilaspekten der Ästhetik und der Haptik variiert.

\subsection{Versuchsteillnehmer}

Zur Ermittlung der benötigten Probandenanzahl für die statistische Aussagefähigkeit wurde eine Vorstudie mit Studenten des Maschinenbaus und der Mechatronik $(n=13)$ durchgeführt. Unter Nutzung der ermittelten Standardabweichung kann die benötigte Mindestprobandenanzahl von $n=22$ für das Konfidenzintervall von $z=95 \%$ berechnet werden. Es werden für die Hauptstudie Anwender ausgewählt, die gewerblich mit Akkuschrauber tätig sind und ein hohes $M a ß$ an Expertise im Umgang mit diesen aufweisen. Das gewährleistet eine differenzierte und möglichst objektive Bewertung. Für die Objektivität in der Durchführung ist zudem wichtig, sämtlichen Probanden ein einheitliches Verständnis der Kriterien zur objektiven Bewertung der Akkuschrauber zu vermitteln. Entsprechend der Ansätze von GERMANN ET AL. werden Schulungsvideos zur Vermittlung des Studieninhalts an die Probanden genutzt. Ebenso werden die Anwendungsfälle, welche im Versuch durch den Probanden durchgeführt werden sollen, aufgezeigt. Ziel dieses Vorgehens ist dabei stets die Erhaltung 
einer hohen Versuchsobjektivität trotz realitätsnaher Verwendung der PowerTools.

\subsection{Anwendungsfälle und Test-Phasen}

Zur Identifikation relevanter Entwicklungsziele ist die Verwendung der Akkuschrauber in realitätsnahen, relevanten Anwendungen nötig. Entsprechend der Arbeiten von GERMANN ET AL. sind für den Test von Akkuschraubern typische Anwendungsfälle das Ein- und Ausschrauben von Holzschrauben, sowie das Holzbohren und Stahlbohren [4]. Durch das Vorgehen wird gewährleistet, dass der Anwender einen umfassenden Einblick in die Funktionalität und Qualität der Geräte während der Versuchsdurchführung erhält. Die Versuchsdurchführung gliedert sich in drei Test-Phasen: 1. Bewertung des Farbeindrucks, 2. Bewertung der Haptik, 3. Bewertung des Farbeindrucks und der Haptik zusammen. Bild 4 bis 6 zeigt die geplante Reihenfolge auf. Aufbauend auf den von KUIJTEVERS ET AL. validierten und standardisierten Fragebogen erfolgt die Erfassung der subjektiven Qualitätsmerkmale [18]. Hierbei werden Fragen zur Ergonomie, Funktionalität und Farbe übernommen und zusätzlich der Aspekt der Attraktivität abgefragt. Die Bewertung erfolgt mittels zustimmungsbasierten 5-stufigen-Likert Skala. Nach vollständigem Abschluss der Versuche wird nach jeder Anwendung des Power-Tools auf einer 10-Stufigen Likert-Skala die wahrgenommene Anwendungseignung jedes Gerätes erfasst.

\section{Phase 1: Farbeindruck}
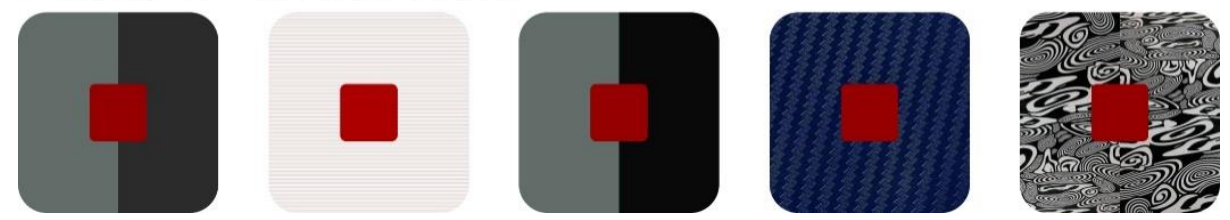

Bild 3 - Farbkombinationen für die verwendeten Power-Tools (links Referenz)

Innerhalb der ersten Phase wird der Farbeindruck mit den bekannten KenngröBen der Designelemente von LEDER UND CARBON und HAN ET AL. variiert: Symmetrie (hohe gegen geringe), Farbkombinationen (hohe gegen geringe Farbdifferenz) und Form (rund gegen eckig) [19, 20]. Die Probanden erhalten jeweils die fünf Farb- und Musterkombinationen auf Karten ausgedruckt und beantworten die zugehörigen Fragen auf dem Fragenbogen. In Bild 3 sind die Farbkarten für die Farben Serie, 3D-Druck, ähnlich zur Serie lackierten Farben, dunkelblaue Farbe mit symmetrischem Muster und kontrastreiche schwarz-weiß Farbkombination mit einem unregelmäßigen Muster abgebildet. 


\section{Phase 2: Haptik}
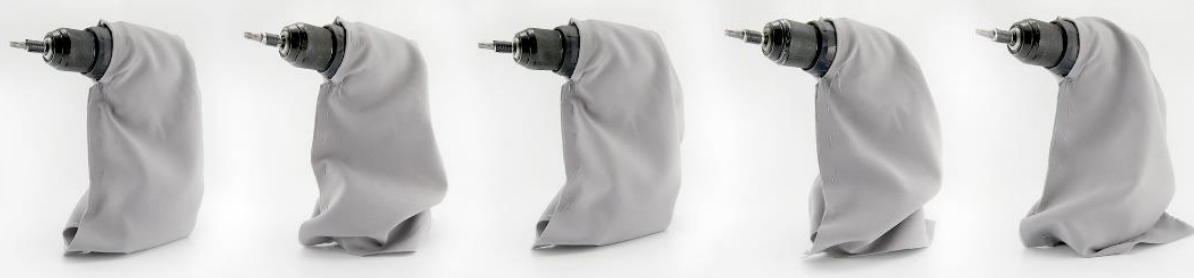

Bild 4 - abgedeckte Akkuschrauber für den objektiven haptischen Eindruck

Für die zweite Phase werden vier Prototypen unterschiedlicher Produktreife, bezogen auf das Gehäuse, erzeugt. Die inneren Komponenten wie Motor, Leistungselektronik und Aktorik sind dabei für alle Prototypen die gleichen. Die Akkuschrauber werden zur Vermeidung von Einflüssen durch den Farbeindruck, wie in Bild 4 dargestellt, den Probanden verdeckt zur Verfügung gestellt. Durch die erfolgten Nacharbeiten wird gezielt Einfluss auf die Haptik des Gehäuses genommen. Dafür verbleibt eines der Prototypen-Gehäuse im additiv hergestellten Rohzustand mit einem $R_{z}$-Wert von $82,80 \mu \mathrm{m}$. Beim zweiten wird die gedruckte Oberfläche lackiert, wodurch ein $\mathrm{R}_{\mathrm{z}}$-Wert von $71,65 \mu \mathrm{m}$ erreicht wird. Das dritte und vierte Gehäuse wird mit unterschiedlichen Farbfolien beklebt. Dadurch werden sehr raue Oberflächen mit $R_{z} 131,48 \mu \mathrm{m}$ und sehr glatte mit $\mathrm{Rz}_{\mathrm{z}}$ 3,88 $\mu \mathrm{m}$ erreicht.

\section{Phase 3: Farbeindruck und Haptik}
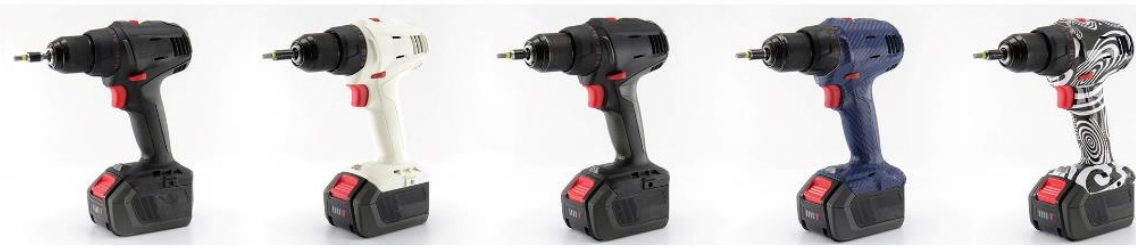

Bild 5 -Akkuschrauber für kombinierten farblichen und haptischen Eindruck

In der dritten Phase wird die Stoffabdeckung abgenommen und der Anwender kann den Akkuschrauber optisch und haptisch kombiniert wahrnehmen und anschließend bewerten. Das additiv hergestellte Gehäuse ist als Prototyp von auBen durch seine schichtweise Herstellung zu erkennen. Das zweite Gehäuse ist in den Serienfarben Grau und Vollschwarz lackiert. Dadurch soll eine Annäherung der Bewertung an die Serie erreicht werden. Der dritte Prototyp ist mit der Farbfolie Blau beklebt mit einem sich symmetrisch wiederholendem Muster. Die dunkle Farbe Blau wurde ausgewählt, da diese sich zusammen mit dem regelmäßig auftretenden Muster visuell nicht so stark auswirkt [21] und so eine objektive Bewertung erreicht wird. Das letzte Gehäuse ist mit in einer kontrastreichen schwarz-weiß Farbfolie mit einem unregelmäßigen Muster beklebt. 
Dadurch wird beim Anwender nach HOGAN ET AL. eine Verwirrung bezüglich der Form und Bewegung erzeugt, wodurch eine negative Beeinflussung erreicht wird [22]. In Bild 5 sind die Farbkombination auf den Akkuschraubern abgebildet.

\section{Zusammenfassung}

Das vorgestellte Forschungsvorhaben hat das Ziel, den Einfluss der Produktreife auf die Bewertung der wahrgenommenen Anwendungseignung zu untersuchen. Hierbei sollen in mehreren Forschungsarbeiten Ansätze entstehen, welche eine Reduktion des Störgrößeneinflusses "Produktreife" ermöglichen. In der Forschung gibt es bislang keine Ansätze um den signifikanten Einfluss des Aspektes der Produktreife innerhalb der Erfassung der subjektiven Bewertung der wahrgenommenen Anwendungseignung zu reduzieren, was dazu führen kann, dass die Produktentwicklung fehlgeleitet wird. Durch das vorgestellte Forschungsdesign und die anhand der Probandenstudien 1 und 2 aufgezeigte Vorgehensweise in der Forschung wird ein strukturiertes Forschungsvorhaben vorgestellt, welches die objektive Erfassung der wahrgenommenen Anwendungseignung erlaubt. Die Erforschung möglicher Einflüsse auf die Bewertung der Produktreife erfolgt dabei am Beispiel der Haptik und Ästhetik. Der aus den Erkenntnissen zu entwickelnde initiale Reduktionsansatz für den Einfluss der Produktreife wird am Beispiel einer Griffgeometrieentwicklung angewandt und validiert. Auf Basis der Forschungsergebnisse soll die Erkenntnis zu relevanten Gestaltmerkmalen aufgebaut werden, die eine gezielte Beeinflussung der wahrgenommenen Produktreife ermöglichen, um so eine frühzeitige Produktvalidierung zu ermöglichen. Damit wird ein Beitrag zur frühen Phase der nutzerzentrierten Produktentwicklung geleistet, in der noch keine Serienprodukte zur Verfügung stehen.

\section{Literatur}

[1] Ponn, J. und Lindemann, U., „Konzeptentwicklung und Gestaltung technischer Produkte", Springer Verlag Berlin Heidelberg, 2008

[2] Kuijt-Evers, L. F. M. et al., "Association between objective and subjective measurements of comfort and discomfort in hand tools", Applied Ergonomics, vol. 38, no. 5, pp. 643-654, 2007

[3] Heine, T., "Additive Manufacturing als Baustein zur gestaltungsgerechten Produktentwicklung in der Fahrzeugelektronik am Beispiel automobiler Zugangssysteme", R. Lachmayer, R.B. Lippert (Hrsg.), Additive Manufacturing Quantifiziert, Springer Verlag Berlin, 2017, pp. 175-194 
[4] Germann, R.; Ribadeneira, E.; Matthiesen, S., "Unbiased Evaluation of Comfort Predictors for the Development of a Handle Geometry", 29th CIRP Design Conference, Póvoa de Varzim, Portugal, 2019

[5] Sauer, J. and Sonderegger, A., "The influence of prototype fidelity and aesthetics of design in usability tests: Effects on user behaviour, subjective evaluation and emotion ", Applied Ergonomics, 40, , 2009, pp. 670677

[6] Virzi, R. A.; Sokolov, J. L.; D., Karis, "Usability Problem Identification Using Both Low- and High-Fidelity Prototypes", Proceedings of the SIGCHI Conference on Human Factors in Computing Systems, NY: ACM, New York, 1996

[7] Jensen, L. S. et al., "Prototyping In Mechatronic Product Development: How Prototype Fidelity Levels Affect User Design Input", Proceedings of the DESIGN 2018 15th International Design Conference, Marjanović, D.; Štorga, M.; Škec, S.; Bojčetić, N.; Pavković, N. , Dubrovnik, Croatia, The Design Society, 2018, pp. 1173-1184

[8] Matthiesen, S. und Germann R., "Ansatz zur objektiven und effizienten Erfassung der empfundenen Anwendungseignung von Power-Tool", in Stuttgarter Symposium für Produktentwicklung SSP 2017, Stuttgart, Deutschland, 2017, S. 395-404

[9] DIN EN ISO 9241-220:2017-05, Ergonomie der Mensch-System-Interaktion - Teil 220: Prozesse zur Ermöglichung, Durchführung und Bewertung menschzentrierter Gestaltung für interaktive Systeme in Herstellerund Betreiberorganisationen (ISO/DIS 9241-220.2:2017)

[10] Kuijt-Evers, L. et al., "Identifying factors of comfort in using hand tools", Applied Ergonomics, 35 (5) , 2004, pp. 453-458

[11] Vink, P. and Hallbeck, S., "Editorial: Comfort and discomfort studies demonstrate the need for a new model," Applied Ergonomics, 43, no. 2, 2012, pp. 271-276

[12] Wall, M. B.; Ulrich, K. T.; Flowers, W. C., "Evaluating prototyping technologies for product design," Research in Engineering Design, vol. 3, no. 3, 1992, pp. 163-177 
[13] Schrage, M., "The Culture(s) of PROTOTYPING," Design Management Journal (Former Series), vol. 4, no. 1, 1993, pp. 55-65

[14] Jensen, L.S.; Öense, A.G.; Mortensen, N.H., "Prototypes in engineering design: Definitions and strategies", Proceedings of the DESIGN 2016 14th International Design Conference, Marjanović, D.; Štorga, M.; Škec, S.; Bojčetić, N.; Pavković, N. , Dubrovnik, Croatia, 2016, The Design Society, Glasgow, 2016, pp. 820-830

[15] Sauer, J.; Seibel, K.; Rüttinger, B., "The influence of user expertise and prototype fidelity in usability tests", Applied ergonomics, 41, no. 1, 2010, pp. $130-140$

[16] Sauer, J. and Sonderegger, A., "The influence of prototype fidelity and aesthetics of design in usability tests: effects on user behaviour, subjective evaluation and emotion", Applied ergonomics, vol. 40, no. 4, 2009, pp. $670-677$

[17] Germann, R.; Jahnke, B.; Matthiesen, S., "Objective usability evaluation of drywall screwdriver under consideration of the user experience", Applied Ergonomics, 75, 2019, S. 170-177

[18] Kuijt-Evers, L. F. M. et al. , "Identifying predictors of comfort and discomfort in using hand tools", Ergonomics, 48, no. 6, 2005, pp. 692-702

[19] Leder, H. and Carbon, C. C., "Dimensions in appreciation of car interior design", Applied Cognitive Psychology, 19, 2005, pp. 603-618

[20] Han, S. H. et al., "Identifying mobile phone design features critical to user satisfaction", Human Factors and Ergonomics in Manufacturing, 14, 2004, pp. 15-29

[21] Ngo, D. C. L.; Teo, L. S.; Byrne, J. G., "Modelling interface aesthetics", Information Sciences, vol. 152, 2003, pp. 25-46

[22] Hogan, B. G.; Cuthill, I. C.; Scott-Samuel, N. E., "Dazzle camouflage, target tracking, and the confusion effect", Behavioral ecology: official journal of the International Society for Behavioral Ecology, vol. 27, no. 5, 2016, pp. 1547-1551 